\title{
Physical insight into the polarization dynamics of semiconductor vertical-cavity lasers
}

\author{
M. P. van Exter, R. F. M. Hendriks, and J. P. Woerdman \\ Huygens Laboratory, Leiden University, P.O. Box 9504, 2300 RA Leiden, The Netherlands
}

(Received 22 July 1997)

\begin{abstract}
The polarization properties of semiconductor vertical-cavity lasers (VCSELs) are generally described with a model introduced by San Miguel, Feng, and Moloney (SFM) in Phys. Rev. A 52, 1728 (1995). We have analyzed this SFM model from an experimentalist's point of view, using the idea that under certain conditions, which are satisfied by most practical VCSELs, the complicated spin dynamics can be adiabatically eliminated, leading to a managable analytical description. We hereby obtain new physical insight and intuitive pictures. One of the key results is the prediction that, via the spin dynamics, the presence of a strong lasing mode with a certain polarization will effectively lead to a broadening and frequency shift of the weak nonlasing mode with orthogonal polarization. This result gives a simple physical explanation for a polarization switch predicted by the model, and leads to further predictions that can be experimentally verified. The analysis also shows how the relaxation oscillations are related to the polarization dynamics and how they might be of crucial importance to experimentally determine the various parameters in the SFM model. We then discuss how the spin elimination reduces the SFM model to existing models for the polarization dynamics of class A (gas) lasers, with intuitive pictures of the polarization evolution on the Poincaré sphere. Finally we will show how, within the context of adiabatic elimination, the cubic crystalline symmetry plays a special role in possible generalizations of the SFM model. [S1050-2947(98)00703-3]

PACS number(s): 42.55.-f
\end{abstract}

\section{INTRODUCTION}

The polarization of semiconductor vertical-cavity surfaceemitting lasers (VCSELs) is an interesting subject to study. The cylindrical symmetry of most designs and the isotropic gain of the cubic material impose no restrictions on the polarization state of the laser. In principle one thus expects these lasers to be indifferent to the polarization direction. The laser might, however, prefer linearly polarized emission over circular or vice versa, as the saturation of the gain can depend on polarization, even when the gain itself is isotropic.

In practice the cylindrical symmetry is broken by all kinds of anisotropies. The most dominant anisotropy has been found to be linear birefringence, caused by (i) stress and strain, acting via the elasto-optic effect [1], and (ii) internal electric fields, acting via the electro-optic effect [2,3]. Application of additional stress, in fact, allows one to manipulate the VCSEL polarization at will, either in a reversible way, via a "hot-spot technique" [1], or in a permanent way, via "local burning", [4]. The electro-optically induced birefringence can be manipulated, at least in optically pumped VCSELs, by varying the doping [2,3]. A coupled-mode description, based on linear birefringence in combination with a generally small amount of linear dichroism (equal to the difference in gain or loss), allows for simple explanations of practically all experimental data [5].

Nevertheless, simple explanations in terms of linear effects are bound to break down somewhere, due to the active nature of the laser and the possible polarization dependence of gain saturation. The simplest and still realistic model to describe the polarization aspects of these nonlinear effects for a semiconductor vertical-cavity laser is a rate equation model introduced by San Miguel, Feng, and Moloney (SFM) [6]. In this model, the active medium is separated in different spin classes, each interacting only with circularly polarized light of one specific handedness. Through saturation the field polarization codetermines the spin population, which then acts back on the field via a spin-dependent gain and refractive index. The SFM model predicts, among others, the possible occurrence of a switch of the output polarization when the pump rate is increased. Polarization switches have indeed been observed experimentally [7-9]. Other predictions concern all kinds of unstable and chaotic behavior for specific situations at high pump rates [10-12], instabilities that have not yet been observed. Alternatively, such polarization switches may result from shifts in the relative tuning of the cavity resonance with respect to the semiconductor gain spectrum, due to self-heating of the device [13]. In a recent experiment, however, switches were in fact observed at constant device temperature, confirming that the nonlinear effects are indeed able to induce polarization switching [14].

Due to the generally complicated spin dynamics, the full SFM model allows only numerical solutions, which limits the amount of physical insight that can be obtained. The influence of this spin dynamics is quantified by the dimensionless parameter $\Gamma$, which is essentially the ratio of the spin-flip rate and the population relaxation rate. In this paper we analyze the SFM model in the regime of fast spin relaxation, or large $\Gamma$, and small to moderate linear anisotropies (see Sec. II for a more precise definition). We will show how the adiabatic elimination of the fast spin dynamics then allows for analytic solutions and how it in fact reduces the polarization dynamics of the laser to that of a class A laser, with a polarization-dependent saturation that reflects the original spin dynamics. The goal of this paper is to get more insight into the SFM model and to obtain simple predictions for the way nonlinear effects will codetermine the VCSELs behavior in practical situations. We specifically look for signatures of weak nonlinear anisotropies in the presence of usually stronger linear anisotropies. The strength of these 
nonlinear signatures proves to be inversely proportional to $\Gamma$; for $\Gamma \rightarrow \infty$ the polarization effects disappear as the optical nonlinearity becomes polarization insensitive.

The crucial parameter $\Gamma$ is not known accurately for practical VCSELs. The first estimates [6] were based on timeresolved photoluminescence experiments with circularly polarized excitation, as found in the literature $[15,16]$. However, it is unclear whether these experiments yield realistic values for $\Gamma$ in practical devices, as photoluminescence experiments are generally performed at low temperature and much lower carrier densities than those encountered in practical devices. In particular in such experiments, the excitonic versus free-carrier aspects will be rather different from those in VCSELs. More realistic estimates of $\Gamma$ should be obtained directly from operating devices. The first results in this category yield $\Gamma>100$, showing that the spin relaxation is indeed very fast $[17,18]$. In hindsight, using the theoretical analysis presented in this paper, this result is consistent with earlier experiments, where the nonlinear anisotropies were observed to be weak [5].

In Sec. II we introduce the SFM model, linearize around the stationary states, and discuss the stability regimes. Also, the relation between the relaxation oscillations and the polarization dynamics is discussed. In Sec. III we show how the linearized equations can be simplified in two different regimes and how this provides physical explanations for the polarization instabilities predicted by the full model. In Sec. IV we return to the original nonlinear equations to show how adiabatic elimination of the fast spin dynamics simplifies the physics. The spin elimination is argued to have a broad validity range; it is shown to reduce the polarization dynamics of a vertical-cavity laser to that of a standard class A (gas) laser. In Sec. V we show how the cubic crystalline symmetry plays a special role in possible generalizations of the SFM model within the spin-adiabatic limit. Finally, Sec. VI contains the summary.

\section{THE SFM MODEL; STATIONARY STATES AND LINEARIZATION}

The field polarization in semiconductor vertical-cavity lasers is generally described by the SFM model, which in ordinary time units reads $[6,10,11]$

$$
\begin{aligned}
\dot{E}_{+}= & -(\epsilon+i \sigma) E_{-}+\kappa(1-i \alpha)[(N-1)+n] E_{+}, \\
\dot{E}_{-}= & -(\epsilon+i \sigma) E_{+}+\kappa(1-i \alpha)[(N-1)-n] E_{-}, \\
\dot{N}= & -\gamma\left[(N-1-\mu)+\left(\left|E_{+}\right|^{2}+\left|E_{-}\right|^{2}\right) N\right. \\
& \left.+\left(\left|E_{+}\right|^{2}-\left|E_{-}\right|^{2}\right) n\right], \\
\dot{n}= & -\gamma_{J} n-\gamma\left[\left(\left|E_{+}\right|^{2}+\left|E_{-}\right|^{2}\right) n\right. \\
& \left.+\left(\left|E_{+}\right|^{2}-\left|E_{-}\right|^{2}\right) N\right] .
\end{aligned}
$$

The notation is as follows: $E_{+}$and $E_{-}$are the circularly polarized components of the (slowly varying) optical field (a factor $e^{-i \omega t}$ has been separated out); $N$ and $n$ are the average inversion between the + and - spin transitions and half the inversion difference, respectively, i.e., the spin-dependent in- version is $N_{ \pm}=N \pm n ; \kappa, \gamma$, and $\gamma_{J}=2 \gamma_{j}+\gamma$ are the decay rates of the optical field, the average inversion, and the inversion difference, respectively; the parameter $\Gamma$, already introduced above, is given by $\Gamma=\gamma_{J} / \gamma ; \mu$ is the normalized pump parameter, being about 0 at threshold (depending on the strength of the dichroism $\epsilon$ ). The optical field is normalized with respect to the saturation field; in an ideal four-level laser $\left|E_{+}\right|^{2}+\left|E_{-}\right|^{2}=1$ corresponds to equal rates of stimulated and spontaneous emission; $\alpha$ is the linewidth enhancement factor, which for most semiconductor lasers is positive, as the refractive index generally decreases with increasing population inversion; $\sigma$ and $\epsilon$ are the linear phase and amplitude anisotropies in angular frequency units. We will mainly concentrate on the case of linear birefringence and linear dichroism in the same direction, i.e., the case where the eigenmodes of the linear problem are linearly polarized. When we choose the $x-y$ coordinate system to coincide with this direction, $\sigma$ and $\epsilon$ are both real valued (the situation $\sigma>0$ and $\epsilon>0$ corresponds with $x$-polarized light having both the highest frequency and highest loss rate). In the more general situation of linear birefringence and dichroism making angles $\phi_{\sigma}$ and $\phi_{\epsilon}$ with respect to the $x$-axis the parameters $\sigma$ and $\epsilon$ become complex, having phase factors $e^{-2 i \phi_{\sigma}}$ and $e^{-2 i \phi_{\epsilon}}$ in Eq. (1a) and $e^{2 i \phi_{\sigma}}$ and $e^{2 i \phi_{\epsilon}}$ in Eq. (1b).

\section{A. Stationary solutions}

The stationary solutions of the above equations have been given in several papers; for the situation of aligned birefringence and dichroism, there are two linearly polarized solutions and two elliptically polarized solution, the latter being stable only in a very limited range of parameter space [1012]. We will limit ourselves to the region of parameter space where only the linearly polarized modes are stable. Without loss of generality we can further limit our treatment to the $x$-polarized state, as the laser feels the difference between $x$ and $y$ only through the sign of $\sigma$ and $\epsilon$; an interchange of the $x$ and $y$ axes is equivalent to a simultaneous sign change of $\sigma$ and $\epsilon$. The stationary $x$ state is easily found to be

$$
E_{+}=E_{-}=Q e^{-i \Delta \omega t},
$$

$$
2 Q^{2}=\frac{\mu-\epsilon / \kappa}{1+\epsilon / \kappa} \approx \mu
$$

$$
N_{\mathrm{eq}}=1+\epsilon / \kappa,
$$

$$
\Delta \omega=\sigma+\alpha \epsilon
$$

Note that, in the presence of (weak) dichroism, the equilibrium inversion $N_{\text {eq }}$ is almost, but not exactly equal to 1 . Via the $\alpha$ factor this results in a small carrier-induced shift of the cavity resonances from the $\left(N_{\mathrm{eq}}=1\right)$ values, which might show up as a shift of the absolute optical frequency when the laser switches from one polarization mode to another. As $|\epsilon| \ll \kappa$ for all practical situations we will frequently use the approximation in Eq. (2b). 


\section{B. Linearization around the $x$-polarized state}

In the spirit of San Miguel et al. [6] we can linearize the original equations around the stationary $x$-polarized state by writing

$$
\begin{gathered}
E_{+}=\left(Q+a_{+}\right) e^{-i \Delta \omega t}, \\
E_{-}=\left(Q+a_{-}\right) e^{-i \Delta \omega t}, \\
S=a_{+}+a_{-}, \\
R=a_{+}-a_{-}, \\
N=N_{\mathrm{eq}}+\Delta N,
\end{gathered}
$$

where $Q$ is real valued and $a_{+}$and $a_{-}$are complex. Linearization of the original equations results in a rigorous separation into two decoupled sets; this helps a lot.

The first set of equations describes the coupled time evolution of the complex optical amplitude of the vector field, as characterized by $S$, and of the average population inversion $N$ :

$$
\begin{aligned}
\dot{S}= & 2 \kappa(1-i \alpha) Q \Delta N, \\
\dot{\Delta} N= & -\gamma\left(1+2 Q^{2}\right) \Delta N \\
& -2 \gamma Q\left(1+\frac{\epsilon}{\kappa}\right) \operatorname{Re}[S] .
\end{aligned}
$$

The second set of equations describes the coupled fluctuations of the polarization direction and ellipticity, characterized by $R$, and of the population difference $n$ :

$$
\begin{aligned}
& \dot{R}=2(\epsilon+i \sigma) R+2 \kappa(1-i \alpha) Q n, \\
& \dot{n}=-\left(\gamma_{J}+2 Q^{2} \gamma\right) n-2 \gamma Q\left(1+\frac{\epsilon}{\kappa}\right) \operatorname{Re}[R] .
\end{aligned}
$$

The three eigenvalues of the first set of equations are easily found. The $(\lambda=0)$ eigenvalue corresponds to phase diffusion. The other two eigenvalues $\lambda=-\lambda_{R} \pm i \omega_{R}$ correspond to the relaxation oscillations in the laser, where

$$
\begin{aligned}
& \lambda_{R}=\frac{\gamma}{2}\left(1+2 Q^{2}\right) \approx \frac{\gamma}{2}(1+\mu), \\
& \omega_{R}=\sqrt{\omega_{0}^{2}-\lambda_{R}^{2}}, \\
& \omega_{0}=2 Q \sqrt{\kappa \gamma(1+\epsilon / \kappa)} \approx \sqrt{2 \kappa \gamma \mu} .
\end{aligned}
$$

We note that a fit of the experimentally observed relaxation oscillation will thus provide for several important laser parameters. This makes it a very powerful experimental tool, in particular, because the value of parameters like $\mu, \kappa$, and $\gamma$ is usually rather uncertain. Specifically, the damping rate $\lambda_{R}$ of the relaxation oscillations at low output power, once corrected for the finite laser linewidth, yields the carrier decay rate $\gamma[19,20]$. The relaxation oscillation frequency $\omega_{R}$ yields the product $\kappa \gamma \mu$. We will find later on that exactly the same product enters the equations for the nonlinear polariza- tion anisotropies. This fortunate result of course reflects the common basis for the intensity and polarization dynamics.

The three eigenvalues of the second set of equations (5), in terms of $R$ and $n$, determine the polarization dynamics. These eigenvalues are more difficult to find, being the roots of the equation

$$
\begin{aligned}
\left(\lambda+\gamma_{J}+\right. & \left.2 Q^{2} \gamma\right)\left[(\lambda-2 \epsilon)^{2}+4 \sigma^{2}\right] \\
& +4 \kappa \gamma Q^{2}(1+\epsilon / \kappa)(\lambda-2 \epsilon+2 \alpha \sigma)=0,
\end{aligned}
$$

which, apart from the sign of $\alpha$, is identical to Eq. (43) in Ref. [12]. When we use the experimental result $|\epsilon| \ll \kappa[5]$ and set $2 Q^{2} \approx \mu$, as in Eq. (2b), the above equation becomes

$\left(\lambda+\gamma_{J}+\gamma \mu\right)\left[(\lambda-2 \epsilon)^{2}+4 \sigma^{2}\right]+2 \kappa \gamma \mu(\lambda-2 \epsilon+2 \alpha \sigma)=0$.

The isotropic case $(\sigma=\epsilon=0)$ has been discussed in [6]. Here the three eigenvalues of Eq. (8) are $\lambda=0$, associated with the diffusion of the polarization direction that arises from the absence of anisotropy, and two others given by

$$
\lambda_{1,2} \approx-\frac{\gamma_{J}}{2} \pm \sqrt{\left(\gamma_{J} / 2\right)^{2}-2 \kappa \gamma \mu}=-\frac{\gamma_{J}}{2} \pm \sqrt{\left(\gamma_{J} / 2\right)^{2}-\omega_{0}^{2}},
$$

where we have used $\gamma \mu \ll \gamma_{J}$. The real or imaginary character of these eigenvalues is governed by the comparison between the spin relaxation rate $\gamma_{J}$ and the relaxation oscillation frequency $\omega_{0}$. We have recently shown the spin relaxation rate $\gamma_{J}$ to be quite large, $\gamma_{J} \approx 200-1000 \mathrm{GHz}$ $[17,18]$. Since the relaxation oscillation frequency is often much smaller at $\omega_{0} \leqslant 2 \pi \times 10 \mathrm{GHz}[21,22]$, we conclude that experiments are generally in the regime $\gamma_{J} \gg 2 \omega_{0}$. In this regime the eigenvalues given above can be approximated as

$$
\begin{aligned}
& \lambda_{1} \approx-\gamma_{J}, \\
& \lambda_{2} \approx-\frac{2 \kappa \mu}{\Gamma} .
\end{aligned}
$$

The related eigenstates show that the eigenvalue $\lambda_{1}$ is associated mainly with a deviation of the inversion difference $n$ from equilibrium, whereas $\lambda_{2}$ is associated with a deviation from linear polarization (the smallness of $\lambda_{2}$ lets SFM conclude that linear polarization is only "marginally stable" [6]). For higher pump parameters $\mu$, the eigenvalue $\left|\lambda_{1}\right|$ decreases and $\left|\lambda_{2}\right|$ increases, until they coincide at $\lambda_{1}=\lambda_{2}=-\gamma_{J} / 2$. For even higher $\mu$, in the experimentally hardly accessible regime $2 \omega_{0}>\gamma_{J}$, the eigenvalues obtain an imaginary part and become each other's complex conjugate. The damped oscillation associated with these eigenvalues is then sometimes denoted as the "polarization relaxation oscillation" [6].

For the more general case, $\sigma \neq 0$ and $\epsilon \neq 0$, exact expressions for the eigenvalues of Eq. (8) are quite complicated, being the roots of a third-order polynomial in $\lambda$. Instead of giving these exact expressions, we concentrate on the condition that one or both linear polarizations are stable, a condition that translates into the requirement that all three eigenvalues correspond to damped motion and thus satisfy 


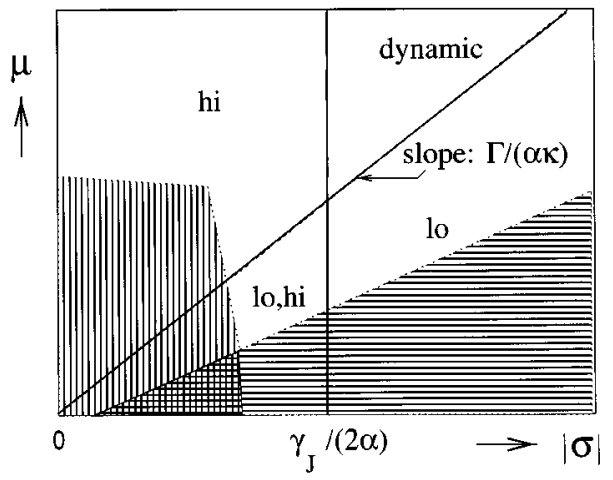

FIG. 1. Stability diagram for the laser polarization in the $(\sigma, \mu)$ plane in the absence of dichroism $(\epsilon=0)$. The stabilities of the lowand high-frequency polarization mode are denoted by the labels "lo" and "hi," respectively. The two solid lines are the stability boundaries. The vertically dashed area shows the parameter range in which adiabatic elimination of the spin dynamics is allowed; the horizontally dashed area shows the range in which nonlinear effects can be treated in a perturbative way.

$\operatorname{Re}(\lambda)<0$. The results of such an analysis can be summarized in a "stability plot," which shows the stability of the low- and high-frequency mode as a function of birefringence $\sigma$ and pump parameter $\mu[10-12]$. As we will need these standard results in the rest of the paper we have presented them as Fig. 1, for $\epsilon=0$, and Figs. 2(a) and 2(b), for $\epsilon=0.02 \sigma$ and $\epsilon=-0.02 \sigma$, respectively. The notation "lo" or "hi" denotes the regime where either the low- or the

(a)

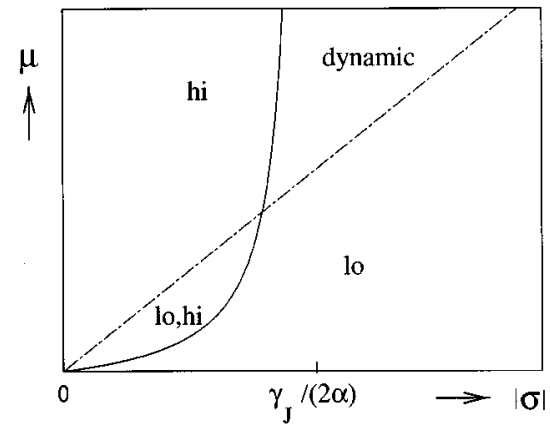

(b)

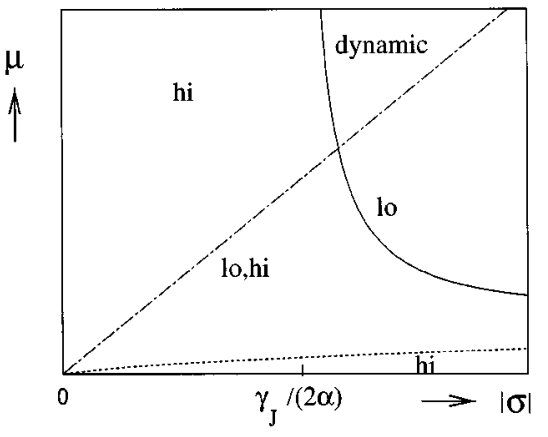

FIG. 2. Stability diagram for the laser polarization in the $(\sigma, \mu)$ plane for the case $\epsilon=0.02 \sigma$ (a) and $\epsilon=-0.02 \sigma$ (b). Note how the stability boundary of the low-frequency mode (sloping line) is hardly affected by the dichroism and how the stability of the highfrequency mode is drastically changed. Note also that at low pump rate only the mode with the lowest linear loss is stable. high-frequency mode is stable; the notation "lo,hi" denotes the regime of bistability where linearization around both the $\sigma<0$ and the $\sigma>0$ mode yields stability; the notation "dynamic" in the upper right-hand part of the figures denotes that at high birefringence and high pump parameter the laser polarization is never stable and that the emitted optical field will show chaotic dynamic behavior $[11,12]$.

The boundaries between the various stability regimes can be found by either setting the $\lambda^{0}$ term in Eq. (8) equal to zero, or by using $\lambda=i \nu$ as a trial solution. For $\epsilon=0$ there are two stability boundaries. The sloping line in Fig. 1 illustrates that the low-frequency mode $(\sigma<0)$ loses its stability at pump parameters above $[10,11]$ :

$$
\mu_{\mathrm{crit}} \approx \frac{\Gamma}{\alpha \kappa}|\sigma| \text {. }
$$

The (almost) vertical line in Fig. 1 shows that the highfrequency mode $(\sigma>0)$ loses its stability for a birefringence larger than $[10,11]$

$$
\sigma_{\mathrm{crit}}=\left(\gamma_{J}+\gamma \mu\right) /(2 \alpha)
$$

A comparison of the two stability boundaries [Eqs. (11) and (12)], using Eq. (6c), shows that they cross at the point where $\omega_{0} \approx \gamma_{J} / \alpha$ (for $\gamma \mu \ll \gamma_{J}$ ). As for semiconductor lasers $\alpha$ is appreciably larger than 1 ; this means that the polarization relaxation oscillations mentioned earlier exist only at pump parameters $\mu$ that lie (high) above this crossing point. For nonzero $|\epsilon| \ll|\sigma|$ the stability boundary for the lowfrequency mode [Eq. (11) is hardly changed, as shown by the sloping lines in Figs. 2(a) and 2(b). The stability boundary for the high-frequency mode is seriously affected though; at high pump parameters it still approximately satisfies Eq. (12), but at low pump parameter it bends away towards low or high birefringence for $\epsilon / \sigma>0$ and $\epsilon / \sigma<0$, respectively. We note that at low pump parameters, when nonlinear effects are relatively unimportant, the only stable mode is obviously the one with the lowest loss, being the low-frequency one for $\epsilon=0.02 \sigma$ and the high-frequency one for $\epsilon=-0.02 \sigma$ [see Figs. 2(a) and 2(b), respectively].

The dashed areas in Fig. 1 show in a schematic way the two regimes in which the complicated polarization dynamics predicted by the SFM model can be treated analytically. The vertically striped area depicts the regime of small linear anisotropies and not too high pump rates $\left(|\sigma|,|\epsilon|, 2 \omega_{0} \ll \gamma_{J}\right)$. In this "adiabatic regime" we will simplify the polarization rate equations [Eqs. (1) and (5)] through adiabatic elimination of the inversion difference $n$. The horizontally striped area depicts the regime of relatively low pump rate and not too low birefringence $[\kappa \mu /(\sigma \Gamma) \ll 1]$, where the linear anisotropies dominate over the nonlinear ones. In this "perturbative regime" we will simplify the equations by treating the nonlinear anisotropies as a relatively weak perturbation. The solutions that we obtain are of course most simple in the "overlap regime" of the two approximations. Although this cross-hatched area looks small in Fig. 1 its can have a large extent in both the $\sigma$ and the $\mu$ direction for the experimental situation of large spin-flip rate, i.e., large $\gamma_{J}$. We believe that most experiments on VCSELs have in fact been performed in this overlap regime, since in the experiment one typically 
has $10 \mathrm{GHz}<|\sigma|<50 \mathrm{GHz},|\epsilon|<3 \mathrm{GHz}, \omega_{0}<60 \mathrm{GHz}$, $\gamma \approx 1 \mathrm{GHz}$, and $\gamma_{J} \approx 200-1000 \mathrm{GHz}[5,17,18]$. In Sec. III we will discuss the simplifications that are possible in the various regimes and give physical reasons for the stability boundaries in Figs. 1, 2(a), and 2(b).

\section{SIMPLIFYING THE LINEARIZED RATE EQUATIONS}

\section{A. The adiabatic regime}

In the adiabatic regime the linearized polarization rate equations [Eqs. (5)] can be simplified through adiabatic elimination of the inversion difference $n(t)$. Inspection of Eq. (5b) shows that this is allowed when the variation of the field polarization, or more specifically $Q \operatorname{Re}[R]$, is slow as compared to the relaxation rate $\gamma_{J}$ of the spin dynamics, which translates into the criterion $|\sigma|,|\epsilon|, 2 \omega_{0} \ll \gamma_{J}$. This corresponds to the vertically striped area in Fig. 1. Adiabatic elimination of $n$, using the obvious inequalities $2 \gamma Q^{2} \ll \gamma_{J}$ and $\epsilon \ll \kappa$, gives

$$
n=-\frac{2 Q}{\Gamma} \operatorname{Re}[R]
$$

Inserting this in Eq. (5a), using $2 Q^{2} \approx \mu$, we obtain the following equation for the polarization fluctuations:

$$
\dot{R}=2(\epsilon+i \sigma) R-\frac{2 \kappa \mu}{\Gamma}(1-i \alpha) \operatorname{Re}[R],
$$

where the first term on the right-hand side represents the linear (cavity-related) anisotropies, whereas the second term, being proportional to the pump parameter $\mu$, represents the nonlinear (medium-related) anisotropy.

The eigenvalues of Eq. (14) are easily found by separating this complex equation into two real-valued equations for $\operatorname{Re}[R]$ and $\operatorname{Im}[R]$. For $\gamma \mu \ll \gamma_{J}$ these eigenvalues are given by the equation

$$
(\lambda-2 \epsilon)^{2}+\frac{2 \kappa \mu}{\Gamma}(\lambda-2 \epsilon)+4 \sigma^{2}+4 \alpha \sigma \frac{\kappa \mu}{\Gamma}=0
$$

with solutions

$$
\lambda_{1,2}=2 \epsilon-\frac{\kappa \mu}{\Gamma} \pm i 2 \sigma \sqrt{1+\alpha \frac{\kappa \mu}{\sigma \Gamma}-\frac{1}{4}\left(\frac{\kappa \mu}{\sigma \Gamma}\right)^{2}}
$$

We note that the same result could have been obtained mathematically by a separation of time scales in Eq. (8); in the adiabatic regime one eigenvalue $\lambda \approx-\gamma_{J}$ is much larger than the other two eigenvalues so that division by $\gamma_{J}$ in combination with the requirement $\left|\lambda_{1,2}\right| \ll \gamma_{J}$ reduces the third-order equation (8) into the second-order equation (15). We also note that the eigenvalues (16) evolve continuously from the $\lambda=0$ and $\lambda=-2 \kappa \mu / \Gamma$ eigenvalues found earlier for the isotropic case.

The important dimensionless parameter in Eq. (16) is the ratio $\kappa \mu /(\sigma \Gamma)=\omega_{0}^{2} /\left(2 \sigma \gamma_{J}\right)$, which quantifies the relative strength of the nonlinear anisotropies as compared to the linear ones. When $\kappa \mu /(\sigma \Gamma) \ll 1$ we end up in the crosshatched "overlap regime", in Fig. 1, where the nonlinear anisotropies act as a small perturbation and where the square root in Eq. (16) can be approximated to give

$$
\lambda_{1,2}=2 \epsilon-\frac{\kappa \mu}{\Gamma} \pm i\left(2 \sigma+\frac{\kappa \mu \alpha}{\Gamma}\right) .
$$

The physical insight comes from the interpretation of the above eigenvalues. These eigenvalues characterize the laser's response to small excursions from the linearly polarized stationary state, excursions that are driven by the random fluctuating force of spontaneous emission. In the optical spectrum of the laser these perpetual excursions are visible as a weak additional spectral peak that differs from the main peak in its polarization and generally also in its optical frequency. This weak peak is often denoted the "nonlasing polarization mode," in contrast to the strong "lasing polarization mode", $[1,4,5]$. Close to threshold (small $\mu$ ) the nonlinear effects are weak and $\lambda_{1,2} \approx 2 \epsilon \pm i 2 \sigma$, so that the frequency splitting between the lasing and nonlasing mode is a direct measure for the linear birefringence $\sigma$, whereas the difference between their spectral widths gives the linear dichroism $\epsilon$. In practice this proves to be a good assumption, making the polarization-resolved optical spectrum a crucial tool to analyze the laser's anisotropies $[1,4,5]$. The above Eq. (17) now shows how the nonlinear interaction in the VCSEL, related to the dynamics of $n$, affects this spectrum and how it gives an additional nonlinear contribution to the damping rate and optical frequency of the nonlasing mode of magnitude

$$
\begin{gathered}
\Delta \gamma_{\text {nonlasing }}=\frac{\kappa \mu}{\Gamma}=\frac{\omega_{0}^{2}}{2 \gamma_{J}}, \\
\Delta \omega_{\text {nonlasing }}=-\alpha \frac{\kappa \mu}{\Gamma}=-\alpha \frac{\omega_{0}^{2}}{2 \gamma_{J}} .
\end{gathered}
$$

This set of equations is a key result of the present paper. Equation (18a) shows how nonlinear effects increase the damping of the nonlasing mode; nonlinear effects can thereby stabilize the lasing mode, even when it has the largest linear loss, and cause phenomena like bistability and polarization switching. Equation (18b) shows that the nonlasing mode is effectively redshifted with respect to the lasing mode. This follows directly from Eq. (17), which shows that the frequency of the polarization beat will increase when the high-frequency mode lases $(\sigma>0)$ and when we linearize around this mode, whereas it will decrease when the lowfrequency mode lases $(\sigma<0)$. In both cases the nonlasing mode is effectively redshifted. Note that by rewriting the "nonlinear damping and frequency shift" in terms of the relaxation oscillation frequency $\omega_{0}$ we have effectively removed all (badly known) device parameters, except for the spin relaxation rate $\gamma_{J}$.

To visualize the origin of the nonlinear contributions to dichroism and birefringence, Fig. 3 gives a graphical representation of the time evolution of the "polarization deviation" $R(t)$ in (a $90^{\circ}$ rotated version of) the complex plane. The linear term $2(\epsilon+i \sigma) R$ in Eq. (14) makes $(\operatorname{Re}[R], \operatorname{Im}[R])$ rotate around the stationary point $(0,0)$, spiraling inward or outward depending on the sign of $\epsilon$. In the meantime the nonlinear term in Eq. (14), depicted as arrows 


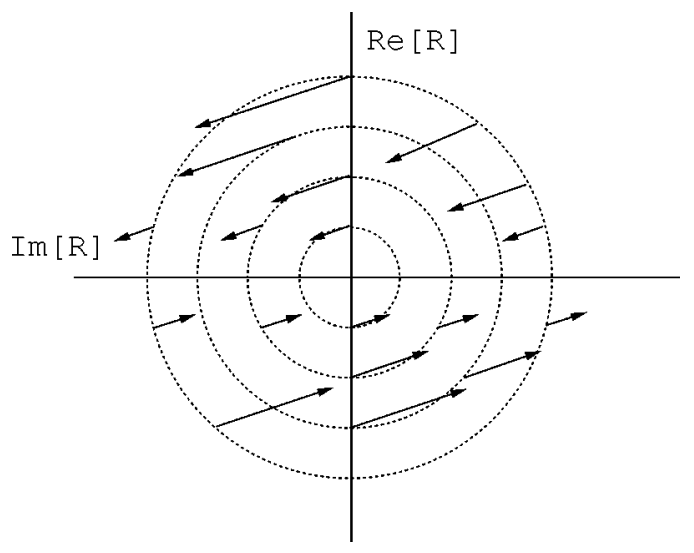

FIG. 3. The evolution around a linearly polarized stationary state, expressed in the deviations $\operatorname{Re}[R]$ and $\operatorname{Im}[R]$. Linear birefringence alone will make $R$ rotate around the stationary point, whereas linear dichroism will push it away or pull it towards this point. The action of the last term in Eq. (14) is presented by the arrows in the figure; they show how the nonlinear anisotropy does not average out over a full round trip, but effectively pulls $R$ towards the stationary state, while also giving it additional spin. The nonlinear term thus acts as effective dichroism and birefringence.

in Fig. 3, will pull $(\operatorname{Re}[R], \operatorname{Im}[R])$ towards the line $(\operatorname{Re}[R]=0)$, while at the same time, for $\alpha>0$, giving it a leftward push above this line and a rightward push below this line. When the nonlinear term is small as compared to the linear one, i.e., in the "overlap regime," its pushing and pulling can be averaged over a full rotation. Averaging then shows how it effectively helps to pull $(\operatorname{Re}[R], \operatorname{Im}[R])$ towards the origin and how it leads to an increase or decrease of the rotation frequency, depending on the sign of $\sigma$ with respect to $\alpha$. Figure 3 thus gives an elegant and intuitive explanation for the effective frequency shift and damping induced by the nonlinear effects, i.e., the mechanism behind Eqs. (18).

The nonlinear contributions to dichroism and birefringence, as predicted by Eqs. (18), give quantitative explanations for the deviations observed in earlier experiments on VCSEL anisotropies [5]. In these experiments linear anisotropies were found to dominate the VCSEL's polarization state and a simple (linear) coupled-mode description was sufficient to explain almost all experimental observations. The small deviations that remained were already tentatively attributed to nonlinear effects [5]. With the theory developed here, we can be more specific. Figure 4 in Ref. [5] indicates that the measured frequency splitting between lasing and nonlasing modes is slightly larger than the linear birefringence in the VCSEL. With the present knowledge we attribute this to a redshift of the nonlasing mode induced by nonlinear effects. The offset of $0.2 \mathrm{GHz}$ mentioned in Ref. [5] is a reasonable value for the redshift that is predicted by Eq. (18b) and the sign agrees with the observation that the high-frequency mode was lasing. Figure 7 in Ref. [5] shows another deviation: the width of the nonlasing mode was found to be larger than could be expected on the basis of the measured linear dichroism, and, although part of this discrepancy could be attributed to the finite width of the lasing mode, about $0.2 \mathrm{GHz}$ remained unexplained. We now attribute this additional damping of the nonlasing mode to non- linear damping and indeed the mentioned value is not unreasonable for the damping predicted by Eq. (18a). This semiquantitative analysis points in the direction of relatively fast spin relaxation $(\Gamma \geqslant 100)$, consistent with recent experiments that address the spin relaxation explicitly $[17,18]$.

\section{B. The perturbative regime}

We will next consider the perturbative regime $[\kappa \mu /(\sigma \Gamma) \ll 1]$, i.e., the horizontally striped area in Fig. 1 . In this regime the nonlinear anisotropies act as a small perturbation to the polarization dynamics and simple expressions for this dynamics can be found even for large linear anisotropies, where the adiabatic elimination of $n$ breaks down. In the perturbative approach we will first neglect the nonlinear term in Eq. (5a), by setting $Q=0$, to obtain

$$
R(t)=R_{0} e^{2(\epsilon+i \sigma) t} .
$$

Substitution of this first-order result into Eq. (5b), using $|\epsilon| \ll|\sigma|, \kappa$ and $\gamma \mu \ll \gamma_{J}$, yields

$$
n(t) \approx-2 Q \gamma \operatorname{Re}\left[\frac{R_{0}}{\gamma_{J}+2 i \sigma} e^{2(\epsilon+i \sigma) t}\right]
$$

We thus find that at relatively large birefringence $\sigma$, where the field polarization varies rapidly in time, $n(t)$ has difficulties following and acquires a phase lag, of the order $2 \sigma / \gamma_{J}$, with respect to $R(t)$. The consequences of this phase lag, and of the nonlinear effects in general, are found by substituting Eq. (20) back into Eq. (5a). When we express the resulting corrections by introducing a time dependence for $R_{0}(t)$ and averaging over a full rotation, using the rotating-wave approximation by keeping only the positive frequency terms, we obtain

$$
\dot{R}_{0}(t) \approx-\frac{\kappa \mu}{\Gamma} \frac{1-i \alpha}{1+2 i \sigma / \gamma_{J}} R_{0}(t)
$$

For $|\sigma| \ll \gamma_{J}$ we recover from Eq. (21) the earlier result Eq. (18) that relatively small nonlinear effects result in an effective dichroism and birefringence of magnitude $\kappa \mu / \Gamma$ and $\alpha \kappa \mu / \Gamma$, respectively; for larger birefringence it shows how the phase lag experienced by $n(t)$ is translated into a redistribution and possible reduction of the nonlinear dichroism and birefringence.

When we do not use the rotating-wave approximation we find a small counter-rotating term (negative frequency), with a magnitude that is determined by the relative strength of the nonlinear anisotropies as compared to that of the linear ones. This term shows that, due to the nonlinear anisotropies, $R(t)$ does not evolve around perfect circles or spirals, but instead around elliptical trajectories. Hoffman and Hess [23] have recently predicted how the counter-rotating term will show up as a, generally very weak, four-wave-mixing peak in the optical spectrum, being a mirror image of the nonlasing peak with respect to the strong lasing peak. Very recently, this peak has been observed experimentally [18]. 


\section{Physical explanations for the stability boundaries}

With the simplified expressions derived above we are now able to give physical explanations for the instability boundaries in Figs. 1, 2(a), and 2(b). The first boundaries to be discussed are the sloping lines in these figures, which show how the low-frequency mode loses its stability at pump parameters above $\mu_{\text {crit }}$. To be more precise, numerical calculations based upon the SFM model predict that when the low-frequency mode lases at low pump parameter, this mode will become elliptically polarized at $\mu_{\text {crit }}$, and lose its stability at slightly higher pump parameter, leading to a polarization switch; when the high-frequency mode initially lases, nothing special occurs $[11,12]$. The explanation we give for this phenomenon is the following: Inspection of Eq. (16), for the practical case $\alpha \gg 1$, shows that when the low-frequency mode lases $(\sigma<0)$, the frequency splitting between the two modes will decrease with increasing pump parameter up to about $\mu_{\text {crit }}$, where the frequencies will coincide. Beyond this point the eigenvalues will quickly acquire large real parts and the polarization becomes unstable. On the other hand, when the high-frequency mode lases $(\sigma>0)$ the nonlinear effects will lead to an increase of the mode splitting and nothing special is expected to happen. A measurement of the polarization frequency splitting versus $\mu$ can thus decide whether the observed polarization switch is induced by nonlinear effects, or by a shift in detuning of the cavity resonance with respect to the semiconductor gain spectrum, as induced by self-heating [13].

In Fig. 3 the instability mentioned above occurs when the nonlinear terms, depicted as arrows, are strong enough to effectively stop the rotation induced by the linear birefringence. The flow pattern around the stationary state will then deviate considerably from the nonsaturated pattern (there will be, among others, a strong counter-rotating term) and at high enough pump rates it will open up to destroy the stability of this state.

The other stability boundaries in Figs. 1, 2(a), and 2(b) show how the high-frequency mode loses its stability at large birefringence. The explanation for these boundaries lies in the breakdown of the validity of the adiabatic elimination of the spin dynamics. For the perturbative regime Eq. (21) already showed how this breakdown leads to a shifted balance between the absorptive and dispersive nonlinear terms. In the graphical representation of Fig. 3 this phase lag corresponds to a rotation of the arrows in a clockwise or counterclockwise way for the high- or the low-frequency mode, respectively. For the case $\epsilon=0$ the effective damping and stabilization of the high-frequency mode $(\sigma>0)$ is thereby lost at values $\sigma>\gamma_{J} /(2 \alpha)$, where the arrows have crossed the horizontal direction and point away from the equator instead of towards it. This inequality agrees with the stability boundary of Eq. (12). For the case $\epsilon \neq 0$ the stability of the highfrequency mode is lost when the damping due to nonlinear effects becomes equal to the linear damping $\epsilon$; this criterion reproduces the curved stability boundaries in Figs. 2(a) and 2(b).

\section{ADIABATIC ELIMINATION OF SPIN DYNAMICS}

To get a broader view on the physics behind the SFM model, and to compare it with existing models for the polar- ization dynamics of gas lasers, we will now return to the original nonlinear equations [Eqs. (1)] and try to solve these via adiabatic elimination. A rigorous adiabatic elimination of both the inversion difference $n$ and the average inversion $N$ ("class A" elimination) has been described in a recent paper by Travagnin et al. [24]. The assumptions used in that elimination are that the optical field fluctuates slowly as compared to both population decay rates $\gamma$ and $\gamma_{J}$, that $\gamma \ll \gamma_{J}$, and that the laser operates close to threshold $\left(\left|E_{+}\right|^{2}+\left|E_{-}\right|^{2} \ll 1\right)$. This leads to a set of rate equations for the fields $E_{+}$and $E_{-}$, which are a polarization variant of third-order Lamb theory [25] and which have as key parameter the ratio of cross- and self-saturation of the circularly polarized fields, for which the expression $[1-(1 / \Gamma)] /[1+(1 / \Gamma)]$ is found [24].

The adiabatic elimination of the average inversion $N$ is disputable, because the relaxation rate $\gamma$ is generally not fast, and in fact often slow, as compared to the time evolution of the optical field. On the other hand, the adiabatic elimination of the inversion difference $n$ from the original rate equations Eqs. (1) is generally allowed. The criterion for this elimination is that the "driving force" in Eq. (1d), i.e., the product $\gamma N\left(\left|E_{+}\right|^{2}-\left|E_{-}\right|^{2}\right)$ varies slowly as compared to the relaxation rate $\gamma_{J}$. Variations in $N$ are intrinsically slow due to the relatively long carrier lifetime $\left(\gamma^{-1} \approx 1 \mathrm{~ns}\right)$, but coupling with the intracavity field via stimulated emission speeds up these fluctuations to the relaxation oscillation frequency $\omega_{0}$, so that the condition for slow variations in $N$ translates into $\omega_{0} \ll \gamma_{J}$. Variations in $\left(\left|E_{+}\right|^{2}-\left|E_{-}\right|^{2}\right)$, i.e., in the elliptical component of the laser field, are also slow under certain conditions. A typical time scale for these ellipticity fluctuations is not the cavity loss rate $\kappa$, but rather a time scale related to polarization anisotropies. When the linear anisotropies dominate over the nonlinear ones, the field polarization evolves approximately with a "frequency" $2(\sigma-i \epsilon)$ [see Eq. (14)], making the condition for adiabatic elimination equivalent to $|\sigma|,|\epsilon| \ll \gamma_{J}$. When the linear anisotropies are so weak that the nonlinear effects dominate, the elimination is also allowed as long as the latter are weak, a condition that corresponds to $\omega_{0} \ll \gamma_{J}$. As discussed in Secs. II and III both conditions, i.e., $|\sigma|,|\epsilon| \ll \gamma_{J}$ and $\omega_{0} \ll \gamma_{J}$, are quite reasonable for practical VCSELs; together they correspond to the vertically striped area in Fig. 1.

Using the inequalities

$$
\begin{aligned}
(N-1) & \ll 1, \\
\left|E_{+}\right|^{2}+\left|E_{-}\right|^{2} & \ll \Gamma
\end{aligned}
$$

in Eq. (1d), the adiabatically eliminated $n$ becomes

$$
n=-\frac{1}{\Gamma}\left(\left|E_{+}\right|^{2}-\left|E_{-}\right|^{2}\right) .
$$

Note that to obtain this result we did not need the assumption of "'operation close to threshold," i.e., $\left|E_{+}\right|^{2}+\left|E_{-}\right|^{2} \ll 1$, as was the case for the full class A elimination, but only the much weaker assumption $\left|E_{+}\right|^{2}+\left|E_{-}\right|^{2} \ll \Gamma$; this assumption is enough to validate the third-order Lamb theory for the field polarization. Note also that the inequality $(N-1) \ll 1$ is 
naturally satisfied in semiconductor lasers where $\kappa \gg \gamma$, and where small deviations from $N=1$ already lead to large variations of the laser field.

At this point it is convenient to separate the optical intensity and polarization by introducing the so-called Stokes vector $\vec{s}=\left(s_{1}, s_{2}, s_{3}\right)$ as $[12,26,27]$

$$
\begin{aligned}
& s_{1}=2 \operatorname{Re}\left[E_{+}^{*} E_{-}\right]=I \cos 2 \chi \cos 2 \phi, \\
& s_{2}=2 \operatorname{Im}\left[E_{+}^{*} E_{-}\right]=I \cos 2 \chi \sin 2 \phi, \\
& s_{3}=\left|E_{+}\right|^{2}-\left|E_{-}\right|^{2}=I \sin 2 \chi,
\end{aligned}
$$

where $I$ is the optical intensity, and where the angles $\chi$ and $\phi$ represent the polarization state. The angle $0 \leqslant \phi \leqslant \pi$ characterizes the polarization direction; it is the angle between the long axis of the polarization ellipse and the $x$ axis. The angle $-\pi / 4 \leqslant \chi \leqslant \pi / 4$ characterizes the ellipticity of the light; $\chi=0$ corresponds to linearly polarized light, whereas $\chi= \pm \pi / 4$ corresponds to circularly polarized light. Using the angles $(2 \chi, 2 \phi)$ as spherical coordinates, the polarization state can be conveniently depicted as a single point on the Poincaré sphere [26].

Substitution of the adiabatic value of $n$ [Eq. (23)] into the original Eqs. (1) yields three equations in terms of $E_{+}, E_{-}$, and $N$. Rewritten in terms of $(N, I, \chi, \phi)$ we get the following spin-eliminated version of the SFM model:

$$
\dot{\chi}=\sigma \sin 2 \phi+\epsilon \sin 2 \chi \cos 2 \phi-(\kappa I / \Gamma) \sin 2 \chi \cos 2 \chi,
$$

$$
\dot{\phi}=-\sigma \frac{\sin 2 \chi}{\cos 2 \chi} \cos 2 \phi+\epsilon \frac{\sin 2 \phi}{\cos 2 \chi}-\alpha(\kappa I / \Gamma) \sin 2 \chi,
$$

$\dot{I}=2 \kappa(N-1) I-2 \epsilon I \cos 2 \chi \cos 2 \phi-2 I(\kappa I / \Gamma) \sin ^{2} 2 \chi$,

$$
\dot{N}=-\gamma\left[(N-1-\mu)+I N-\frac{I^{2}}{\Gamma} \sin ^{2} 2 \chi\right],
$$

which is identical to Eqs. (34a), (34c), and (34d) in the erratum of Ref. [11], if the population difference $d$ is eliminated there.

A further adiabatic elimination of the average inversion $N$ would obviously reduce the above set of equations to a special case of the class A (gas) laser [28]. However, even without the elimination of $N$, the first two equations [Eqs. (25a),(25b)], which describe the polarization evolution of a spin-eliminated VCSEL, are already equivalent to those of a class A laser. The mathematical argument is that the average inversion $N$ simply does not appear in these equations. The physical argument is that a deviation of $N$ from equilibrium will lead to equal gain or loss for all Stokes parameters and thus cannot affect the field polarization.

When comparing the polarization dynamics of a spineliminated VCSEL with that of a general class A gas laser, we note that the VCSEL is a special case because of the specific form of its nonlinear anisotropies, which is determined by $\Gamma$, as a measure for the polarization anisotropy of the saturation, and $\alpha$, as a measure for the dispersive nature of the saturation. In Eqs. (25a) and (25b) the linear anisotropies are of course also a special case as we restricted the treatment to linear birefringence and dichroism in the same direction. However, because of the mentioned equivalence, we can now use the result that Van Haeringen [28] found for the general class A (gas) laser to obtain the polarization rate equations for a spin-eliminated VCSEL with arbitrary linear and circular birefringence and dichroism. By rewriting Van Haeringen's anisotropy functions $h_{1}(\chi, \phi)$ and $h_{2}(\chi, \phi)$ in our notation we find

$$
\begin{aligned}
\dot{\chi}= & \sigma \sin 2\left(\phi-\phi_{\sigma}\right)+\epsilon \sin 2 \chi \cos 2\left(\phi-\phi_{\epsilon}\right)+\epsilon_{c} \cos 2 \chi \\
& -(\kappa I / \Gamma) \sin 2 \chi \cos 2 \chi, \\
\dot{\phi}= & -\sigma \frac{\sin 2 \chi}{\cos 2 \chi} \cos 2\left(\phi-\phi_{\sigma}\right)+\epsilon \frac{\sin 2\left(\phi-\phi_{\epsilon}\right)}{\cos 2 \chi}+\sigma_{c} \\
& -\alpha(\kappa I / \Gamma) \sin 2 \chi,
\end{aligned}
$$

where the angles $\phi_{\sigma}$ and $\phi_{\epsilon}$ denote the orientation of the linear birefringence and linear dichroism with respect to the $x$ axis, and where the symbols $\sigma_{c}$ and $\epsilon_{c}$ are possible circular birefringence and dichroism, respectively.

As the polarization rate equations of a spin-eliminated vertical-cavity VCSEL and a class A (gas) laser are identical, within the context of third-order Lamb theory, this should in principle allow for an easy comparison between the two lasers. In practice, the polarization dynamics might still be quite different as the lasers operate in a different parameter regime of the same set of equations. First of all, the ideal isotropic gas laser generally shows a strong preference for either linear or circular emission, whereas the ideal isotropic VCSEL is expected to show only a mild preference for linearly polarized emission, due to the relatively large spin flip rate; for gas lasers typically $\Gamma<10$, whereas VCSELs have $\Gamma>100[17,18]$. As a consequence, Van Haeringen [28] has to work with the adiabatic intensity $I(\chi, \phi)$, which might depend strongly on $\chi$ and $\phi$, where for VCSELs one is often allowed to use a constant intensity $I$. Secondly, the gain spectrum of gas lasers generally has a symmetric Voigt-type profile, whereas that of semiconductor lasers is highly asymmetric. As a consequence, the gain maximum in the gas corresponds to zero detuning, whereas that in the semiconductor still contains "intrinsic detuning" that is reflected in a nonzero $\alpha$ factor and that breaks the symmetry between low and high optical frequencies.

To obtain some more insight into the polarization rate equations (25a) and (25b) we will present them graphically in terms of flow lines on the Poincare sphere [29,30]. Figure 4 gives graphical representations of the action of the four driving mechanisms that can be distinguished in these rate equations. Figure 4(a) shows how the combined linear and circular birefringence alone will make the Stokes vector $\vec{s}$ rotate at a uniform rate $|\vec{\Omega}|$ around the vector $\vec{\Omega}=\left(\sigma \cos 2 \phi_{\sigma}, \sigma \sin 2 \phi_{\sigma}, \sigma_{c}\right)$. Figure 4(b) shows how the combined linear and circular dichroism makes the Stokes vector $\vec{s}$ decay towards the vector $\left(\epsilon \cos 2 \phi_{\epsilon}, \epsilon \sin 2 \phi_{\epsilon}, \epsilon_{c}\right)$. Figure 4(c) shows how the "absorptive" nonlinear effect, i.e., the effect that is independent of $\alpha$, will pull the Stokes vector $\vec{s}$ towards the equator with a force proportional to the optical intensity $I$ multiplied by $\sin 4 \chi$, i.e., twice the devia- 

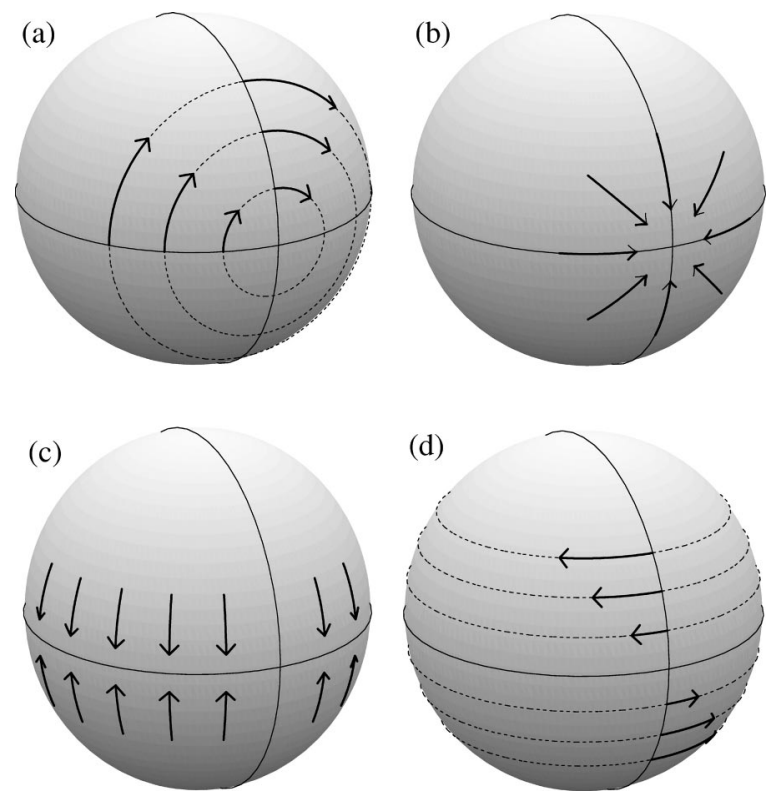

FIG. 4. Graphical representation on the Poincare sphere of the polarization evolution due to the four driving mechanisms: (a) only birefringence $(\sigma<0)$, (b) only dichroism $(\epsilon<0)$, (c) only " absorptive" nonlinear effects (for $\alpha=0$ ), (d) only "dispersive" nonlinear effects (effect of $\alpha \neq 0$ only).

tion from the equator for small $\chi$. Figure $4(\mathrm{~d})$ shows how the "dispersive" nonlinear effect, i.e., the one that scales with $\alpha$, causes a rotation around the north pole with a strength that once more scales with the product $I \sin 4 \chi$.

Whereas the action of the individual driving mechanisms is easily understandable in the graphical representation of Fig. 4, it is their combined action that makes the physics interesting. As a first example of interesting behavior we mention the case where the nonlinear effects are fully isotropic $(\Gamma \rightarrow \infty)$, making the last term in Eqs. (26a) and (26b) equal to zero. For this simple case, the combined action of just linear birefringence and dichroism in different directions already makes the stationary states elliptically polarized; it in fact produces two elliptical eigenstates with the same handedness, as also follows from a linear coupled-mode description [6]. As a second example we mention the case of aligned linear birefringence and linear dichroism that forms the heart of this paper. For small deviations from the linearly polarized stationary state this case was described in Sec. III with Eq. (14) and graphically represented as Fig. 3. We now understand that Fig. 3 is just a head-on view of the Poincaré sphere (Fig. 4), where $\operatorname{Re}[R]$ and $\operatorname{Im}[R]$ correspond to $\chi$ and $-\phi$, respectively, while the line $(\operatorname{Re}[R]=0)$ corresponds with the equator of that sphere.

\section{ROLE OF CRYSTALLINE SYMMETRY}

In the previous sections we have shown how adiabatic elimination of the inversion difference $n$ reduces the polarization dynamics of a VCSEL to that of a class A laser. A similar elimination will be possible for other fast variables that are introduced to the problem, as, for instance, a further separation of the spin populations in electrons and holes. If one retains the rotational symmetry of the saturation process the introduction of these new variables will then produce no new physics, but only lead to a different value and a somewhat different interpretation of $\Gamma$. The reason is that for a class A laser with rotation-symmetric saturation one needs only one parameter $\Gamma$ to describe the polarization dependence of the saturation; this parameter is essentially the ratio of self-saturation and cross saturation of the circularly polarized modes, or the ratio of the saturation power for circularly polarized light as compared to that for linearly polarized light. Within the adiabatic limit, the only way to really generalize the SFM model is therefore the removal of the rotational symmetry of the saturation process by the introduction of the cubic crystalline symmetry of the III-V semiconductor material on which most VCSELs are based. The hard way to introduce the crystalline axes into the problem is by extending the microscopic "atomic'" four-level model of SFM into a full band structure, i.e., by performing an extensive calculation of the $\vec{k}$-dependent densities of states and transition moments [31]. The easy way is to eliminate these microscopic variables from the start and to restrict the discussion to the symmetry of the macroscopic problem.

To discuss the symmetry we will follow a general approach in nonlinear optics in which the dynamic response of the active medium is completely contained in the frequency dependence of the third-order susceptibility tensor [32,33]

$$
\chi_{i j k l}^{(3)}\left(\omega_{1}+\omega_{2}+\omega_{3} ; \omega_{1}, \omega_{2}, \omega_{3}\right)
$$

where $i, j, k, l$ are Cartesian indices, being either $x, y$, or $z$. To describe the laser dynamics with this formalism, one needs to known the frequency behavior of the $\chi^{(3)}$ tensor for optical frequencies, i.e., in the neighborhood of the point $\omega_{1} \approx \omega_{2} \approx-\omega_{3} \approx \omega_{\text {laser }}$. Symmetry imposes strong restrictions on the number of independent coefficients of the 81element $\chi^{(3)}$ tensor. Most textbooks on nonlinear optics contain tables that show the number of independent elements of the above tensor to be only 4 in cubic crystalline media and 3 in rotation-symmetric media (see, e.g., [32]) For the special case $\omega_{1}=\omega_{2}=-\omega_{3}$ the number of independent elements reduces even further to 3 and 2, respectively. For an isotropic gain medium the two independent elements are essentially the saturation power for circularly polarized light and that for linearly polarized light. The ratio of these two is the only parameter that determines the laser's polarization dynamics; it is basically the parameter $\Gamma$ of the SFM model, or the parameter $S$ of Van Haeringen. Only for a nonrotationally symmetric cubic crystalline gain medium will there be an additional (third) degree of freedom left, being the coefficient $A$ that describes the "cubic" $[1+A \cos (4 \phi)]$ angle dependence of the saturation power for linearly polarized light.

Whether or not one is allowed to limit the treatment in terms of $\chi^{(3)}$ to the point $\omega_{1}=\omega_{2}=-\omega_{3}$, thereby reducing the system to a class A laser, depends on the frequency dependence of $\chi^{(3)}$ as compared to the various optical frequencies that play a role. The answer to this question is generally different for the isotropic and anisotropic component of $\chi^{(3)}$, which describe the polarization-independent and polarization-dependent parts of the saturation, respectively. The frequency dependence of the former reflects the dynamics of the overall inversion of the active medium and as the latter is slow, the isotropic part of $\chi^{(3)}$ will vary strongly 
with frequency. One is therefore not allowed to treat the isotropic saturation, and related intensity fluctuations, in terms of a simple class A model. The anisotropic part of $\chi^{(3)}$, on the other hand, varies much less with frequency, due to the fast response of the active medium to polarization changes. For the anisotropic part of $\chi^{(3)}$ one might therefore just as well use only the value at the exact point $\omega_{1}=\omega_{2}=-\omega_{3}$, at least when the relevant optical frequencies differ much less than the inverse response time of the medium to polarization changes. This will then (again) reduce the polarization rate equations of the VCSEL to those of a class A laser.

We note that the symmetry argument given above does not only apply to a bulk piece of cubic crystalline material, but also to any vertical-cavity structure engineered from this material, when the overall device symmetry is $\overline{4} \mathrm{~m}$. It is thus in particular valid for quantum well VCSELs grown on a (100) substrate, which is by far the most common case. Again, in these lasers the polarization-dependent saturation can be fully described by one or two parameters, depending on whether one assumes rotation symmetric saturation or includes the cubic crystalline symmetry in the form of an orientation-dependent saturation power. The fact that in practice the exact symmetry of the laser is broken by anisotropies, like the linear birefringence mentioned in the Introduction, will hardly change this saturation behavior as these anisotropies are generally very small $\left(\ll 10^{-4}\right.$ in terms of the complex refractive index).

\section{SUMMARY}

The key results obtained in this theoretical overview of VCSEL polarization are as follows. Through adiabatic elimination of the spin or inversion difference $n$ we have derived simple expressions for the nonlinear contribution to the measured spectrum of the lasing and nonlasing mode. The validity range of these expressions is quite large; they remain valid as long as the optical field varies slowly as compared to the medium response to polarization changes, which translates into $|\sigma|,|\epsilon|, 2 \omega_{0} \ll \gamma_{J}$, i.e., to the vertically striped area in Fig. 1, where $\sigma$ and $\epsilon$ are the linear birefringence and dichroism, respectively, $\omega_{0}$ is the relaxation oscillation frequency, and $\gamma_{J}$ is related to the spin-flip rate. A further restriction to the case where the linear anisotropies dominate over the nonlinear ones, i.e., the cross-hatched area in Fig. 1, provides the result that is most useful for current experiments. Equations (18) show how nonlinear effects are predicted to result in (i) an extra damping of the nonlasing mode by an amount $\mu \kappa / \Gamma$, and (ii) a redshift of the frequency of the nonlasing mode by an amount $\alpha(\mu \kappa / \Gamma)$. We have argued that most experiments are indeed performed in this "overlap regime", as typical values for the various parameters are $10 \mathrm{GHz}<|\sigma|<50 \mathrm{GHz},|\epsilon| \leqslant 3 \mathrm{GHz}[6], \omega_{0} \leqslant 60$ $\mathrm{GHz}[21,22]$, and $\gamma_{J} \approx 200-1000 \mathrm{GHz}[17,18]$. To facilitate the comparison between theory and experiment we propose to study the relaxation oscillations, as these should provide for an easy experimental approach to quantify several important laser parameters.

We have discussed several experiments in which the effective frequency shift and broadening, which are predicted to arise from the nonlinear effects, have been observed or will be observable $[5,18]$. We have also discussed the physical origin of the two types of polarization instabilities of VCSELs. One of these instabilities was attributed to the effective redshift of the nonlasing mode, making the frequency of this mode, at some pump rate, overlap with that of the lasing mode. The second instability could be associated with a breakdown of the validity of the adiabatic elimination of the spin dynamics.

We have shown how the "SFM model'" for semiconductor lasers reduces to the "Van Haeringen model" for gas lasers, when the population inversion is adiabatically eliminated. The polarization rate equations of the two models were found to be already fully equivalent when only the population difference $n$ is eliminated. This allows for an easy comparison between polarization effects in VCSELs and gas lasers.

Finally, we have shown how the cubic crystalline symmetry plays a special role in possible generalizations of the SFM model within the context of adiabatic spin elimination. Keeping the rotation symmetry of the saturation, there proves to be only one parameter that describes the polarization dependence of the saturation process, being the parameter $\Gamma$ in the SFM model and the ratio of self- and crosssaturation of the circularly polarized modes in the class A model. Possible generalizations can therefore only affect the value and interpretation of this parameter. Within the context of adiabatic elimination, the only way to introduce a new parameter into the problem is to explicitly account for the cubic crystalline symmetry since this breaks the rotation symmetry of the saturation.

For completeness we add that our (and SFM's) model of a VCSEL assumes that the device is spatially and spectrally uniform, i.e., effects due to spatial and spectral hole burning are neglected. Although so far the model has been quite successful we suspect that these complications will ultimately limit its validity.

Note added in proof. After completion of this work the nonlinear redshift and damping given by Eqs. (18a) and (18b) were also discussed in Ref. [34].

\section{ACKNOWLEDGEMENT}

We thank M. San Miguel and J. Martin-Regalado for useful discussions. We further acknowledge the support of the Stichting voor Fundamenteel Onderzoek der Materie (FOM), the European Union ESPRIT Project No. 20029 (ACQUIRE), and the TMR network Grant Nos. ERB4061 and PL951021 (Microlasers and Cavity QED).
[1] A. K. Jansen van Doorn, M. P. van Exter, and J. P. Woerdman, Appl. Phys. Lett. 69, 1041 (1996).

[2] M. P. van Exter, A. K. Jansen van Doorn, and J. P. Woerdman, Phys. Rev. A 56, 845 (1997).
[3] R. F. M. Hendriks, M. P. van Exter, J. P. Woerdman, L. Weegels, A. van Geelen, K. H. Gulden, and M. Moser, Appl. Phys. Lett. 71, 2599 (1997).

[4] A. K. Jansen van Doorn, M. P. van Exter, and J. P. Woerdman, 
Appl. Phys. Lett. 69, 3635 (1996).

[5] A. K. Jansen van Doorn, M. P. van Exter, A. M. van der Lee, and J. P. Woerdman, Phys. Rev. A 55, 1473 (1997).

[6] M. San Miguel, Q. Feng, and J. V. Moloney, Phys. Rev. A 52, 1728 (1995).

[7] C. J. Chang-Hasnain, J. P. Harbison, L. T. Florez, and N. G. Stoffel, Electron. Lett. 27, 163 (1991).

[8] S. Jiang, Z. Pan, M. Dagenais, R. A. Morgan, and K. Kojima, Appl. Phys. Lett. 63, 3545 (1993).

[9] H. Kawaguchi, I. S. Hidayat, Y. Takahashi, and Y. Yamayoshi, Electron. Lett. 31, 109 (1995).

[10] J. Martin-Regalado, M. San Miguel, N. B. Abraham, and F. Prati, Opt. Lett. 21, 351 (1996).

[11] M. Travagnin, M. P. van Exter, A. K. Jansen van Doorn, and J. P. Woerdman, Phys. Rev. A 54, 1647 (1996); 55, 4641(E) (1997).

[12] J. Martin-Regalado, F. Prati, M. San Miguel, and N. B. Abraham, IEEE J. Quantum Electron. 33, 765 (1997).

[13] K. D. Choquette, R. P. Schneider Jr., K. L. Lear, and R. E. Leibenguth, IEEE J. Sel. Top. Quantum Electron. 1, 661 (1995).

[14] J. Martin-Regalado, J. L. A. Chilla, J. J. Rocca, and P. Brusenbach, Appl. Phys. Lett. (to be published).

[15] T. C. Damen, L. Vina, J. E. Cunningham, J. Shah, and L. J. Sham, Phys. Rev. Lett. 67, 3432 (1991).

[16] S. Bar-Ad and I. Bar-Joseph, Phys. Rev. Lett. 68, 349 (1992).

[17] R. F. M. Hendriks, M. P. van Exter, J. P. Woerdman, K. H. Gulden, and M. Moser (unpublished).
[18] M. P. van Exter, A. Al-Remawi, and J. P. Woerdman (unpublished).

[19] G. P. Agrawal and N. K. Dutta, Long-Wavelength Semiconductor Lasers (Van Nostrand-Reinhold, New York, 1986), p. 244.

[20] M. P. van Exter, W. A. Hamel, J. P. Woerdman, and B. R. P. Zeijlmans, IEEE J. Quantum Electron. 28, 1470 (1992).

[21] D. Kuksenkov, S. Feld, C. Wilmsen, H. Temkin, S. Swirhun, and R. Leibenguth, Appl. Phys. Lett. 66, 277 (1995).

[22] H. Li, IEEE Photonics Technol. Lett. 8, 1594 (1996).

[23] H. F. Hofmann and O. Hess (unpublished).

[24] M. Travagnin, M. P. van Exter, and J. P. Woerdman, Phys. Rev. A 56, 1497 (1997).

[25] M. Sargent III, M. O. Scully, and W. E. Lamb, Jr., Laser Physics (Addison-Wesley, Reading, MA, 1974).

[26] O. S. Heavens and R. W. Ditchburn, Insight into Optics (Wiley, Chichester, 1991).

[27] R. Guenther, Modern Optics (Wiley, New York, 1990).

[28] W. Van Haeringen, Phys. Rev. 158, 256 (1967).

[29] H. de Lang, D. Polder, and W. van Haeringen, Philips Tech. Rev. 32, 190 (1971).

[30] M. V. Tratnik and J. E. Sipe, J. Opt. Soc. Am. B 2, 1690 (1985).

[31] L. Fratta, F. Prati, and M. Travagnin (unpublished).

[32] P. N. Butcher and D. Cotter, The Elements of Nonlinear Optics (Cambridge University Press, Cambridge, 1990).

[33] R. W. Hellwarth, Progress in Quantum Electronics (Pergamon Press, New York, 1977), Vol. 5, p. 1.

[34] H. van der Lem and D. Lenstra, Opt. Lett. 22, 1698 (1997). 